\title{
El COVID-19 y la salud de los jóvenes. Tres preguntas a la psiquiatra Liliana Alva*
}

\section{COVID-19 AND THE HEALTH OF YOUNG PEOPLE. THREE QUESTIONS TO THE PSYCHIATRIST LILIANA ALVA}

\footnotetext{
Luciana Agostini

Universidad de Lima

lagostin@ulima.edu.pe

Jorge Florián

Universidad de Lima

jflorian@ulima.edu.pe
}

Johnny Gutiérrez

Universidad de Lima
jgutierr@ulima.edu.pe

La cuarentena impuso no solamente restricciones de circulación, sino también la imposibilidad de mantener interrelaciones físicas laborales, académicas y amicales. Esta última es de vital importancia para los jóvenes, dada la etapa en la que se encuentran, lo que puede traer como consecuencia diversas afectaciones emocionales. La psiquiatra Liliana Alva responde con brevedad y precisión tres preguntas sobre la manera en que ha impactado la pandemia en la vida de los adolescentes.

Según su experiencia clínica, durante este periodo pandémico, ¿cuáles son los trastornos emocionales que se presentan con mayor frecuencia, la gravedad de ellos y los rangos de edad en los que más aparecen? ¿Qué otros factores se identifican en la prevalencia?, ¿se puede hablar de que se activan predisposiciones?, ¿o estos casos ya venían en aumento?

Los problemas emocionales que he observado en la práctica clínica durante este periodo están referidos a los problemas de estrés agudo y adaptativo que tienen relación con la pandemia y el COVID-19; casos nuevos y descompensación de depresión; trastornos del espectro ansioso como ansiedad generalizada, trastorno de pánico y en menor proporción trastorno obsesivo compulsivo de ideas de contaminación, así como compulsiones de limpieza o aseo personal. También pacientes con problemas de regulación emocional diagnosticados con trastornos de personalidad, e intentos de suicidio. La gravedad de los trastornos depende de factores como pertenecer a población

\footnotetext{
* Médica cirujana egresada de la Universidad Ricardo Palma, médica psiquiatra egresada de la Universidad Nacional Mayor de San Marcos, psiquiatra asistente en el Departamento de Promoción de la Salud Mental del Hospital Hermilio Valdizán, miembro del Equipo de Telesalud del Hospital Hermilio Valdizán. Tiene formación psicoterapéutica en terapia dialéctica conductual. Estudios de maestría en Salud Pública.
} 
vulnerable a presentaciones severas del COVID-19, infectarse, sobrevivir o tener un duelo por el COVID-19; o por las consecuencias psicosociales propias de las medidas de salud pública impuestas para contener la pandemia como el desempleo, trabajo y estudio remoto, distanciamiento social de redes de soporte como familia y amistades, existencia previa de problemas psicosociales como violencia o algún trastorno mental antes de la pandemia. Existen estudios del impacto en la pandemia que revelan que las mujeres, adultos mayores, niños y adolescentes son grupos vulnerables al efecto negativo en la salud mental.

La situación de la salud mental en el Perú era crítica antes de la pandemia; los trastornos mentales fueron la primera causa de años de vida saludable (AVISA) perdidos (668 114 AVISA, $12,7 \%$ del total), lo que genera principalmente discapacidad; y encabezando la lista se encontraba la depresión. Por tanto, podemos inferir que estos trastornos ya eran un problema de salud pública antes de la aparición del COVID-19 y durante la pandemia - que sacó a flote las carencias de nuestro sistema de salud- se hicieron evidentes las necesidades no cubiertas de la población en el área de la salud mental.

Recientemente la Facultad de Psicología y Ciencias de la Educación de la Universidad de Cataluña (2021), así como otras instituciones dedicadas al estudio de la salud, han presentado un investigación sobre las consecuencias del COVID-19 en la salud mental de las personas, y se ha determinado que los colectivos con más riesgo de sufrir los efectos psicológicos de esta enfermedad son las mujeres, el personal de primera línea de atención, las personas dedicadas al cuidado de personas enfermas y los jóvenes. A partir de esta introducción, le solicitamos que nos responda las siguientes preguntas: ¿qué consecuencias psicológicas sufren los jóvenes después de haber pasado por un proceso de infección por el COVID-19? ¿Existen ciertos trastornos psicológicos o grupos de riesgo que puedan verse más afectados por las circunstancias generadas por la pandemia? ¿En qué medida se ve influido el rendimiento académico de un joven estudiante que pasa por este tipo de situaciones? Sabiendo que cada caso es especial y que cada persona o joven tiene sus propias características y respuestas frente a la enfermedad, ¿cuál sería el tratamiento más adecuado desde un punto de vista multidisciplinario para mejorar la salud mental de estas personas?

Las consecuencias psicológicas están relacionadas con la experiencia de la enfermedad. Aquellos que padecen formas graves del COVID-19 podrían presentar trastornos de estrés postraumático, trastorno de pánico, insomnio, depresión por el aislamiento y el estigma de estar enfermos o poder contagiar, o emociones relacionadas con la culpa, o a causa de consecuencias en el área laboral y académica.

Sí existen personas o grupos de riesgo que se verán más afectados; están aquellos con trastornos mentales graves como esquizofrenia o trastorno bipolar, quienes, además de 
sufrir el estrés propio de la coyuntura, vieron afectada la continuidad de sus controles y atenciones. Asimismo, como ya mencioné antes, las personas con problemas de depresión, ansiedad y trastornos de personalidad relacionados con la tolerancia a la frustración y consumo de sustancias. No es parte del tema en discusión, pero el grupo de adultos mayores, por la dificultad de migrar digitalmente para mantener interacción con sus pares, también es un grupo muy afectado; lo mismo su contraparte, el grupo de niños, adolescentes y adultos jóvenes, quienes dejaron de relacionarse e interactuar con sus pares.

Los problemas emocionales que acompañan a la depresión y ansiedad afectan la capacidad de concentración, atención, motivación; conductualmente se muestran menos productivos y esto se ve reforzado con pensamientos propios de estos estados emocionales como los de minusvalía: "No sirvo" o "No soy bueno para esto", ideas de desesperanza: "Ya para qué hago esto si las cosas no cambiarán", ideas catastróficas motivadas por el miedo presente de la coyuntura de pandemia. Lo mencionado repercute en el rendimiento académico de quien lo padece, ya que, como se mencionó, se afectan capacidades indispensables para el aprendizaje y cumplimiento de las tareas asignadas.

El abordaje integral de los problemas emocionales y psicosociales implica el trabajo de un equipo multidisciplinario capacitado en consejería, detección temprana y diagnóstico de trastornos mentales y problemas psicosociales, tratamiento psicoterapéutico o farmacológico de ser necesario, y la intervención familiar o de grupos de apoyo para el fortalecimiento de las redes de apoyo del individuo. Los equipos de salud mental muchas veces están integrados por psiquiatras, psicólogos, médicos de familia, profesionales en enfermería y trabajo social con competencias en salud mental; y en la medida de lo posible, una vez detectado un caso y derivado oportunamente a un especialista en salud mental, se puede actuar tempranamente en el abordaje integral del joven.

Teniendo en cuenta los puntos mencionados anteriormente, ¿qué recomendaciones se le podría dar a un joven que está lidiando con las consecuencias psicológicas de haberse contagiado del COVID-19? ¿Y qué sugerencias se podría dar a las personas para enfrentar las consecuencias de este proceso de confinamiento social generado por la pandemia?

Las recomendaciones en ambos casos van desde mantener medidas de bioseguridad personal, ya que los jóvenes son la población que se vacunará al final en nuestro medio; hasta realizar, en la medida de lo posible, las actividades que disfrutaba hacer antes de enfermarse 0 antes de la pandemia; en el caso del deporte o actividades al aire libre, con medidas de distanciamiento y uso de mascarilla. Asimismo, continuar con sus actividades de higiene personal, clases virtuales, trabajo, tratar de apoyarse emocionalmente y mantener comunicación con la familia y amistades cercanas, puesto que en 
situaciones complejas emocionales es necesario dejarse apoyar por su círculo cercano; entrenarse en técnicas de respiración diafragmática, conciencia plena y relajación muscular; evitar información excesiva sobre la pandemia y de sitios web no oficiales, porque el riesgo de encontrar información falsa podría generar mayor angustia. $Y$ buscar ayuda especializada si las consecuencias psicológicas generan un deterioro significativo de la funcionalidad, ya sea académica, social, familiar o laboral. 